\title{
Development of Low Endotoxin Gelatin for Regenerative Medicine
}

\author{
Yoshitaka Kanayama, * Chizuru Aoki, and Yasuo SaKaI \\ Central Research Institute, Jellice Co., Ltd.; 4-1-4 Wakabayashi, Wakabayashi-ku, Sendai, Miyagi 984-0826, Japan. \\ Received September 21, 2006; accepted November 18, 2006; published online November 24, 2006
}

\begin{abstract}
The basic concept of tissue engineering, an actively applied technology in regenerative medicine, is the coordination between cells, scaffolds, and proliferation factors. Collagen and gelatin are materials that have mostly been used as scaffolds because of their excellent affinity for cells, they are biodegradable, and their ability to form gel. However, very few of the collagen or gelatin preparations used as tissue engineering material have been prepared with low levels of endotoxin, which may trigger fever, shock and a fall in blood pressure even in very minute quantities. Therefore we decided to decrease the content of endotoxin in gelatin so that this biomaterial is not harmful to the body but still maintains its properties, namely, bioaffinity and biodegradation for use in the fields of cell culture and regenerative medicine. In this paper, we describe a method whereby endotoxin could be removed from gelatin solution by using an ultrafiltration membrane with a molecular weight cut-off of $100 \mathrm{kDa}$ and low endotoxin gelatin, endotoxin activity in $0.1 \%$ gelatin solution was below $0.03 \mathrm{EU} / \mathrm{ml}$, was obtained as the resulting filtrate solution.
\end{abstract}

Key words collagen; gelatin; endotoxin; cell culture; scaffold

Collagen accounts for about a third of the structural proteins of the body and it is present in the skin, blood vessels, organs, bone and connective tissue. Collagen also accounts for approximately $70 \%$ of the dermal structural ingredient of skin and it is the primary ingredient of the extracellular matrix surrounding the outer membrane of the cell body (scaffold) which supports the cell population.

Gelatin on the other hand is collagen with a triple helical structure that has lost its $\gamma$ and $\beta$ chains following the application of heat, and is also called heat-denatured collagen. Gelatin is used not only in food but also in basic ingredients used in cosmetic products and as a base material of soft and hard capsules used in the preparation of medicinal products. Gelatin like collagen is widely used as biomaterial that forms the scaffold supporting cell populations and is also widely used to coat the surfaces of sterile containers facilitating cell attachment and improving the ability of cells to proliferate. In medical practice, collagen and gelatin are used as biomaterials that promote the regeneration of true skin-like tissue in injuries affecting the whole layer of skin.

However, there are no set standards for the endotoxin levels of gelatin which is listed in the Japanese Pharmacopoeia and which is also widely used as a material for medical products. The primary ingredient of endotoxin is lipopolysaccharide (LPS) which is a conserved component of the outer membrane of gram-negative bacteria. LPS is constantly being released in bacterial surroundings similar to the constant shedding of the horny layer of human skin. When bacteria undergo auto-destruction, all the endotoxin is released from the bacteria. When LPS invades the body, even a minute amount can trigger fever, shock, a fall in blood pressure and in worst cases may cause death. ${ }^{1,2)}$ There are also several reports that endotoxin damages various types of cells $^{3-6)}$ suggesting that this substance must be eliminated as much as possible from raw materials that are to be used as biomaterials in regenerative medicine. Endotoxin may be inactivated by heating it to a high temperature of $250^{\circ} \mathrm{C}$ for $60 \mathrm{~min}$ or more ${ }^{7)}$ ruling out autoclaving at $121^{\circ} \mathrm{C}$ which is the setting used for sterilization.

In general, distillation, reverse osmosis, absorption, mo- lecular weight cut-off and ultrafiltration (UF) using a membrane of several $\mathrm{kDa}$ etc., are the methods most commonly used for removing endotoxin from solutions such as water. However, for various reasons, it is difficult to remove endotoxin from collagen and gelatin solutions. For example, with the distillation method, it is possible to completely remove the endotoxin in water; however, it is impossible to isolate the endotoxin in high-molecular weight protein solutions. When gelatin is subjected to continuous heat of a high temperature, it turns brown, and the quality is extremely decreased making its application impossible. With reverse osmosis, endotoxin is removed together with high molecular proteins such as gelatin and so its application is also impossible. In addition, there is a procedure which uses an absorbent, nylon 66 membrane, that is exposed to zeta potential and endotoxin is filtered by absorption. However, gelatin solutions have a higher ionic strength than that of water and this therefore affects the removal of endotoxin. Thus, an effective rate of removal as is the case with water is difficult. On the other hand, the UF procedure is used as the method of choice for removing endotoxin from water and solutions with low molecular-weight ingredients as in the case of injectable drugs that are highly controlled for endotoxin levels because they are administered directly into blood vessels. Use of an UF membrane makes it possible to remove harmful endotoxin from the filtrate while low molecular-weight substances such as antibiotics, salts, sugars and low molecular weight proteins remain. However, the molecular weight cut-off of the UF membrane that is being used is about several $\mathrm{kDa}$, and so proteins with a molecular weight of approximately $10 \mathrm{kDa}$ or higher are removed. As such, this procedure can only be used for the removal of substances in low molecularweight solutions. Due to that, there are no procedures that use UF to remove endotoxin from solutions of gelatin that are high molecular weight proteins of approximately 50 $100 \mathrm{kDa}$.

In this paper, we report a method using UF membrane with an appropriate molecular weight cut-off for the removal of endotoxin from gelatin solutions to produce low endotoxin gelatin of a higher quality which meets the standards for "pu- 
rified gelatin" in the Japanese Pharmacopoeia.

\section{MATERIALS AND METHODS}

Gelatin Gelatin (Jellice, Sendai, Japan) that was prepared by acid extraction from fresh pig skin was used. ${ }^{8)}$

Preparation of Low Endotoxin Gelatin Acid-processed pig skin gelatin was prepared to $10 \%(\mathrm{w} / \mathrm{w})$ using purified water, and allowed to adequately swell at room temperature. After it had completely disintegrated while shaking in a $60{ }^{\circ} \mathrm{C}$ water bath, the temperature was kept at $50^{\circ} \mathrm{C}$. After passing the solution through a polyethylsulfone membrane filter (Advantec Toyo Kaisha, Japan) of pore size $0.2 \mu \mathrm{m}$, it was filtered with an UF system (PELLICON-2 Mini Filter, Millipore Corporation, U.S.A.) equipped with an UF membrane of a molecular weight cut-off of 300,100 or $30 \mathrm{kDa}$. The filtrate obtained was packed unmodified and transported in a sealed sterilized endotoxin-free bag. The amount of endotoxin in the gelatin solution was measured using the limulus amebocyte lysate assay with a sensitivity of $0.03 \mathrm{EU} / \mathrm{ml}$. The result was rated negative only when the endotoxin activity in $0.1 \%$ gelatin solution was below $0.03 \mathrm{EU} / \mathrm{ml}$.

High Performance Liquid Chromatography (HPLC) The HPLC system used to analyze the molecular weight distribution of the gelatin was LC10A VP (Shimadzu, Tokyo, Japan), and the column $(\times 2)$ was Asahipak GS-620 $(7.5 \mathrm{~mm}$ ID $\times 500 \mathrm{~mm}$ ) (Showa Denko K.K., Japan). The measurements were performed at an absorbance of $214 \mathrm{~nm}$ and a temperature of $50^{\circ} \mathrm{C}$. The eluting solutions were $50 \mathrm{~mm}$ $\mathrm{KH}_{2} \mathrm{PO}_{4}$ and $50 \mathrm{~mm} \mathrm{Na} \mathrm{HPO}_{4}(\mathrm{pH}$ 6.8). The analysis time was $60 \mathrm{~min}$ and flow rate $1 \mathrm{ml} / \mathrm{min}$.

Jelly Strength The gelatin solution was prepared to $6.67 \%(\mathrm{w} / \mathrm{w})$ and to the concentration that was predetermined according to the experimental conditions. The solution was placed in a jelly measuring cup (internal diameter $60 \mathrm{~mm}$, and height $60 \mathrm{~mm}$ ). After standing it in a $10^{\circ} \mathrm{C}$ water bath for $16-18 \mathrm{~h}$, the jelly strength was measured using Texture Analyzer TA-XY2i (Stable Micro System Ltd., U.K.). ${ }^{9)}$

Viscosity A $6.67 \%(\mathrm{w} / \mathrm{w})$ strength of the gelatin solution was prepared to a set concentration that met the experimental conditions. The solution was then placed in a specified cell and kept in a $39^{\circ} \mathrm{C}$ water bath to stabilize. Viscosity was then measured using a small oscillating viscosimeter $(A \& D$ Co., Ltd., Japan).

Preparation of the Sponge A 1.0\% low endotoxin gelatin solution was whipped using a hand mixer and immediately placed in a freezer pre-set to $-80^{\circ} \mathrm{C}$. After injecting the solution into a freezer-cooled stainless steel container to freeze thoroughly, it was lyophilized.

Endotoxin Test This test was performed by the limulus amebocyte lysate assay. Pyrogen-free water (Japanese Pharmacopoeia "water for injection"; Otsuka Seiyaku, Japan) was used for all the water used for dilution and endotoxin-free water (Et (-) water). The reagent used in the limulus amebocyte lysate assay was PYROGENT 03 Plus (Cambrex Bio Science Walkesville Inc., U.S.A.). First, $5.2 \mathrm{ml}$ of Et (-) water was slowly added to the packed limulus amebocyte lysate (LAL reagent) and mixed to dissolve without whipping. Aliquots of $100 \mu \mathrm{l}$ of the diluted LAL reagent was injected into endotoxin-free test tubes $(12 \times 75 \mathrm{~mm})$ dry-heat sterilized at $250^{\circ} \mathrm{C}$. A similarly sterilized endotoxin-free cap was immediately placed and the mixture kept frozen at $-80^{\circ} \mathrm{C}$. In addition, Et $(-)$ water was added such that the titer of the $E$. coli endotoxin vial, which is a standard product in PYROGENT 03 Plus became $20 \mathrm{EU} / \mathrm{ml}$. The mixture was vigorously shaken for $15 \mathrm{~min}$ in a test tube shaker to prepare the stock solution.

Next, at the time of use, Et (-) water was used to dilute the stock solution to prepare $0.2 \mathrm{EU} / \mathrm{ml}$ of the standard endotoxin solution. Et $(-)$ water (negative control), endotoxin standard solution, and $100 \mu \mathrm{l}$ aliquots of the various dilutions of each sample were respectively added to the test tubes containing the LAL reagent that had been thawed at room temperature. The various mixtures were incubated at $37 \pm 1{ }^{\circ} \mathrm{C}$. Precautions were taken such that there was no impact to the test tubes during the incubation. After $60 \mathrm{~min}$, each test tube was quickly inverted $180^{\circ}$ and the condition of the gelation observed.

Antigenicity Test Antigenicity of the low endotoxin gelatin was measured by ELISA. First, in order to prepare a gelatin-sensitive ball, $100 \mathrm{ml}$ of a $10 \mu \mathrm{g} / \mathrm{ml}$ gelatin solution was prepared using $25 \mathrm{~mm}$ phosphate buffer solution (PBS) containing $0.15 \mathrm{~m} \mathrm{NaCl}$. An ELISA ball (Sumitomo Bakelite; Tokyo, Japan) was placed in a $300 \mathrm{ml}$ capped bottle and half of the gelation solution mentioned above was added, and shaken for $>60 \mathrm{~min}$ using a rotor. The rest of the gelatin solution was added and again similarly shaken. At the end of the shaking, the gelatin solution in the bottle was discarded and PBS added to wash the ball. After discarding the washings, an appropriate amount of PBS (BSA-PBS) containing $0.1 \%$ of bovine serum albumin, $0.005 \%$ Tween 20 and $0.05 \%$ Proclin 300 was added and the mixture shaken overnight using a rotor. The following day, the BSA-PBS was discarded and the ball washed several times with PBS and BSAPBS. This was used as the gelatin-sensitive ball.

Next, $200 \mu \mathrm{l}$ aliquots of the prepared specimen that was serially diluted with BSA-PBS were injected into plastic test tubes. To these test tubes was added $100 \mu \mathrm{l}$ aliquots of guinea pig anti-gelatin antiserum (primary antibody) that had been diluted 1000-fold. Furthermore, as a negative control, instead of the antibody and primary antibody, $300 \mu \mathrm{l} \mathrm{BSA-}$ PBS was added to a test tube, and as a positive control, instead of the specimen, $200 \mu \mathrm{l}$ of BSA-PBS was added to a test tube to which $100 \mu$ l of the primary antibody had been added. The prepared test tubes were well shaken and stood for $10 \mathrm{~min}$ at room temperature. One gelatin sensitive ball was added to each test tube and stood for $30 \mathrm{~min}$ in a $37^{\circ} \mathrm{C}$ water bath. After that, the gelatin sensitive ball was washed with PBS, and goat anti-guinea pig IgG antibody (secondary antibody) that was labeled with horseradish peroxidase diluted 600 -fold was added and stood for $>30 \mathrm{~min}$ in a $37^{\circ} \mathrm{C}$ water bath. At the time of use, the gelatin-sensitive ball washed with PBS was transferred to a fresh test tube pre-injected with $500 \mu \mathrm{l}$ of $0.2 \% o$-phenylendiamine- $\mathrm{HCl}$ in $50 \mathrm{~mm}$ citric buffer ( $\mathrm{pH} 5.9$ ) containing $0.02 \% \mathrm{H}_{2} \mathrm{O}_{2}$, and stood for $30 \mathrm{~min}$ in a $37^{\circ} \mathrm{C}$ water bath. To each test tube was added $1500 \mu \mathrm{l}$ of $1 \mathrm{~N} \mathrm{H}_{2} \mathrm{SO}_{4}$ to stop the enzyme reaction, and the 492 absorbance measured within $60 \mathrm{~min}$. The binding rate of each specimen was determined taking that of the positive control taken as $100 \%$, and that of the negative control as $0 \%$. 


\section{RESULTS}

Gelatin solution with a mean molecular weight of $50 \mathrm{kDa}$ was processed using an UF system packed with an UF membrane with a molecular weight cut-off of 300,100 or $30 \mathrm{kDa}$ in accordance with the preparation method for low endotoxin gelatin that is documented in the "Materials and Methods". The activity of endotoxin in the acquired filtrate was measured by LAL assay with a sensitivity of $0.03 \mathrm{EU} / \mathrm{ml}$. Only when the endotoxin activity in a $0.1 \%$ gelatin solution was below $0.03 \mathrm{EU} / \mathrm{ml}$ that the activity was rated as negative. Thus, filtrates penetrating UF membranes with molecular weight cut-off of 100 or $30 \mathrm{kDa}$ were rated as negative, but those which penetrated one with a molecular weight cut-off of $300 \mathrm{kDa}$ were rated as positive. Furthermore, the endotoxin activity of unprocessed gelatin was measured in the same study and the results indicated that the level of activity reached 1000-fold that of low endotoxin (Table 1). The same measurements were performed several times to confirm reproducibility, but the results remained unchanged. When an UF membrane of molecular weight cut-off of $300 \mathrm{kDa}$ was used for the processing, the gelatin obtained had an endotoxin activity below $0.3 \mathrm{EU} / \mathrm{ml}$ demonstrating that low endotoxin gelatin could not be prepared using this size membrane. This however, confirmed that the use of an UF membrane with a molecular weight cut-off of $100 \mathrm{kDa}$ enabled removal of endotoxin to a level close to that of low endotoxin gelatin. Therefore, an UF procedure using an UF membrane of appropriate molecular weight cut-off may be considered an effective method for removing endotoxin in gelatin.

An important property necessary for gelatin to be used as scaffold in the field of regenerative medicine is its ability to gel. So, after diluting the acquired low endotoxin gelatin to $2 \%$, it was stood for $18 \mathrm{~h}$ at $4{ }^{\circ} \mathrm{C}$ to determine whether it would gel. We found that low endotoxin gelatin obtained from gelatin solution that had penetrated the UF membrane with a molecular weight cut-off of $100 \mathrm{kDa}$ had the ability to gel, but that obtained from gelatin solution that had penetrated the membrane with a molecular weight cut-off of $30 \mathrm{kDa}$ did not. Additionally, we found that both the gelatin which had penetrated the membrane with a molecular weight cut-off of $300 \mathrm{kDa}$ (not low endotoxin gelatin) and unprocessed gelatin also had the ability to gel (Table 1).

From the results to date, when gelatin with a mean molecular weight of approximately $50 \mathrm{kDa}$ was used, the filtrate that was obtained after it had penetrated an UF membrane with a molecular weight cut-off of $100 \mathrm{kDa}$ was low endotoxin gelatin, which gels under the previously mentioned conditions. In order to solve the phenomenon of gelatin loss when unprocessed gelatin is made to penetrate an UF membrane with a molecular weight cut-off of $30 \mathrm{kDa}$, the molecular weight distribution of gelatin solution before and after it had penetrated the UF membrane was analyzed by HPLC. The results indicate that the ingredients of a high molecular weight of $>50 \mathrm{kDa}$ were significantly decreased when the UF membrane is penetrated (Fig. 1) and that gelation was difficult to achieve suggesting there was a low amount of gelatin with a molecular weight of $30-40 \mathrm{kDa}$ or higher. On the other hand, comparison studies of gelatin solutions with the ability to gel and that had penetrated UF membranes of molecular weight cut-off of 300 and $100 \mathrm{kDa}$, indicated that
Table 1. Changes in Endotoxin Activity and Gelation Ability before and after Ultrafiltration

\begin{tabular}{|c|c|c|c|}
\hline & $\begin{array}{l}\text { Molecular weight } \\
\text { cut off of } \\
\text { ultrafiltration } \\
\text { membrane } \\
(\mathrm{kDa})\end{array}$ & $\begin{array}{l}\text { Endotoxin } \\
\text { rating } \\
(\mathrm{EU} / \mathrm{ml})\end{array}$ & $\begin{array}{c}\text { Gelation } \\
\text { ability } \\
(\bigcirc / \times)\end{array}$ \\
\hline \multirow{4}{*}{$\begin{array}{l}\text { Gelatin } \\
\text { (mean molecular } \\
\text { weight } 50 \mathrm{kDa} \text { ) }\end{array}$} & - & $\begin{array}{c}\text { Positive } \\
(30-300)\end{array}$ & $\bigcirc$ \\
\hline & 30 & $\begin{array}{l}\text { Negative } \\
(<0.03)\end{array}$ & $\times$ \\
\hline & 100 & $\begin{array}{l}\text { Negative } \\
(<0.03)\end{array}$ & 0 \\
\hline & 300 & $\begin{array}{c}\text { Positive } \\
(0.03-0.3)\end{array}$ & 0 \\
\hline \multirow{4}{*}{$\begin{array}{l}\text { Gelatin } \\
\text { (mean molecular } \\
\text { weight } 100 \mathrm{kDa} \text { ) }\end{array}$} & - & $\begin{array}{c}\text { Positive } \\
(30-300)\end{array}$ & 0 \\
\hline & 30 & - & - \\
\hline & 100 & $\begin{array}{c}\text { Negative } \\
(<0.03)\end{array}$ & $\bigcirc$ \\
\hline & 300 & $\begin{array}{l}\text { Positive } \\
(0.03-0.3)\end{array}$ & 0 \\
\hline
\end{tabular}

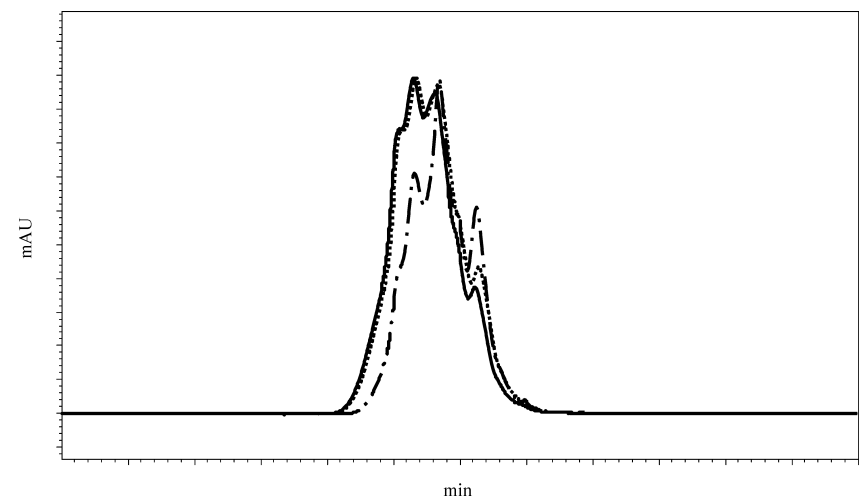

Fig. 1. Changes in Molecular Weight Distribution of Gelatin with Mean Molecular Weight of Approximately $50 \mathrm{kDa}$

-; unprocessed gelatin, ...; molecular weight cut off $300 \mathrm{kDa}$, post-penetration, ---; molecular weight cut off $100 \mathrm{kDa}$ post-penetration,.$--\cdot ;$; molecular weight cut off $30 \mathrm{kDa}$ post-penetration.

there was no change in the molecular weight distribution (Fig. 1).

Furthermore, we investigated whether we could produce low endotoxin gelatin that gels by processing gelatin with a mean molecular weight of approximately $100 \mathrm{kDa}$, through the same UF membrane with a molecular weight cut-off of $100 \mathrm{kDa}$ that had produced the low endotoxin gelatin which gels. Our results demonstrated that when the molecular weight distributions before and after the processing were compared, the high molecular weight ingredients of $>100$ $\mathrm{kDa}$ were decreased but there was still strong gelation with the filtrate obtained after the processing (Fig. 2). In addition, the results of the endotoxin test confirmed that it was a low endotoxin gelatin (Table 1). Moreover, we had confirmed that low endotoxin gelatin with the ability to gel may be similarly prepared even when gelatin of a mean molecular weight of approximately $100 \mathrm{kDa}$ was used. We also found that gelatin solution of a mean molecular weight of approximately $100 \mathrm{kDa}$ could not penetrate an UF membrane with a molecular weight cut-off of $30 \mathrm{kDa}$. Furthermore, we have demonstrated that filtrate with the ability to gel and that which 
could penetrate an UF membrane with a molecular weight cut-off of $300 \mathrm{kDa}$ was achievable, but the endotoxin activity was rated as positive demonstrating that low endotoxin gelatin could not be obtained (Table 1).

From the above findings, it became evident that by using an UF system packed with an UF membrane with a molecular weight cut-off of approximately $100 \mathrm{kDa}$, low endotoxin gelatin that gels may be prepared from gelatin with a mean molecular weight of approximately $50-100 \mathrm{kDa}$. The basic property as gelatin was confirmed by measuring jelly strength and viscosity. The jelly strength (gelatin concentration of $2.0 \%$ ) of low endotoxin gelatin obtained from gelatin with a mean molecular weight of approximately $50 \mathrm{kDa}$ was $15.1 \mathrm{~g}$, while that obtained from gelatin with a mean molecular weight of approximately $100 \mathrm{kDa}$ was $51.5 \mathrm{~g}$. On other hand, viscosity (gelatin concentration of $2.0 \%, 39.0^{\circ} \mathrm{C}$ ) of the former and latter was 1.8 and $2.7 \mathrm{mPa} \cdot \mathrm{s}$, respectively.

We also found that the acquired low endotoxin gelatin showed good results with regards to antigenicity. When a comparison was made based on specimen concentration in $50 \%$ binding rate, the antigenicity of the low endotoxin gelatin was a tenth or less that of the original gelatin, confirming the low antigenicity of the product. Commercially available

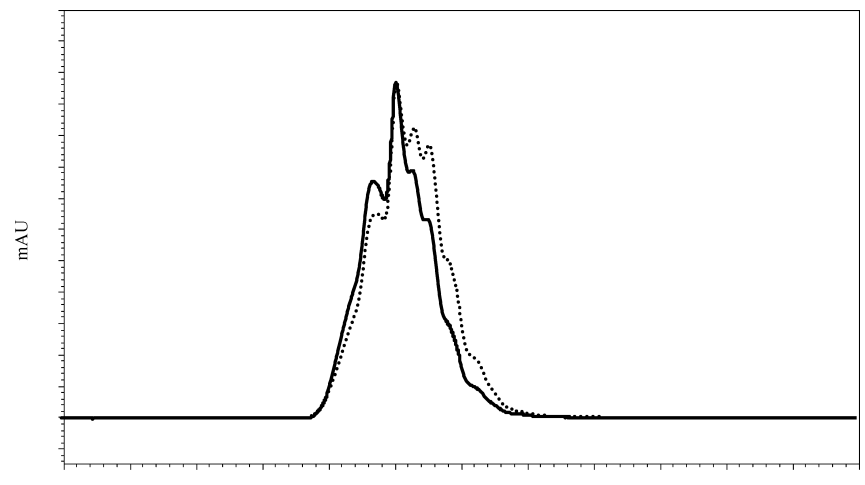

$\min$

Fig. 2. Changes in Molecular Weight Distribution of Gelatin with Mean Molecular Weight of Approximately $100 \mathrm{kDa}$

-; unprocessed gelatin, $\cdots$; molecular weight cut off $100 \mathrm{kDa}$. collagen has low antigenicity, making this product very appealing to use. This property is acquired when the telopeptide of collagen is digested following protease treatment thereby converting the collagen to atelo-collagen. This decreases the antigenicity. When the antigenicity of low endotoxin gelatin was compared with that of commercially available collagen, it had equivalent or less antigenicity (Fig. 3). The results obtained by using the above mentioned method demonstrate that we were successful in producing low endotoxin gelatin that gels and that it had similar properties to conventional gelatin. Additionally, this gelatin had significantly lower endotoxin content and low antigenicity.

The morphology of gelatin that is used as scaffold in regenerative medicine is more porous compared to most forms of jelly. Therefore, whether this porous form can be obtained is an important point. Thus, we prepared a gelatin sponge using the low endotoxin gelatin that was actually obtained. The sponge had elasticity, and microscopic examination of a section showed a porous morphology (Fig. 4). We could only prepare a small-sized gelatin sponge, however, in a factory with processing technology, it will be possible to produce a sponge with a size, hardness, elasticity, biodegradability, pore diameter etc. that is more suitable for regenerative medicine.

\section{DISCUSSION}

We set out to produce low endotoxin gelatin which met the Japanese Pharmacopoeia standards for "purified gelatin" as gelatin is being used as scaffold in regenerative medicine. Our method for the production of low endotoxin gelatin will permit the supply of high quality biomaterial which is being demanded in the fields of cell culture and regenerative medicine.

In this method, we used an UF system that was packed with a membrane having a molecular weight cut-off of $100 \mathrm{kDa}$ since gelatin has a molecular weight of 50 $100 \mathrm{kDa}$, and low endotoxin gelatin was obtained as the filtrate. The low endotoxin gelatin produced had equivalent or less antigenicity when compared to collagen, making it ap-

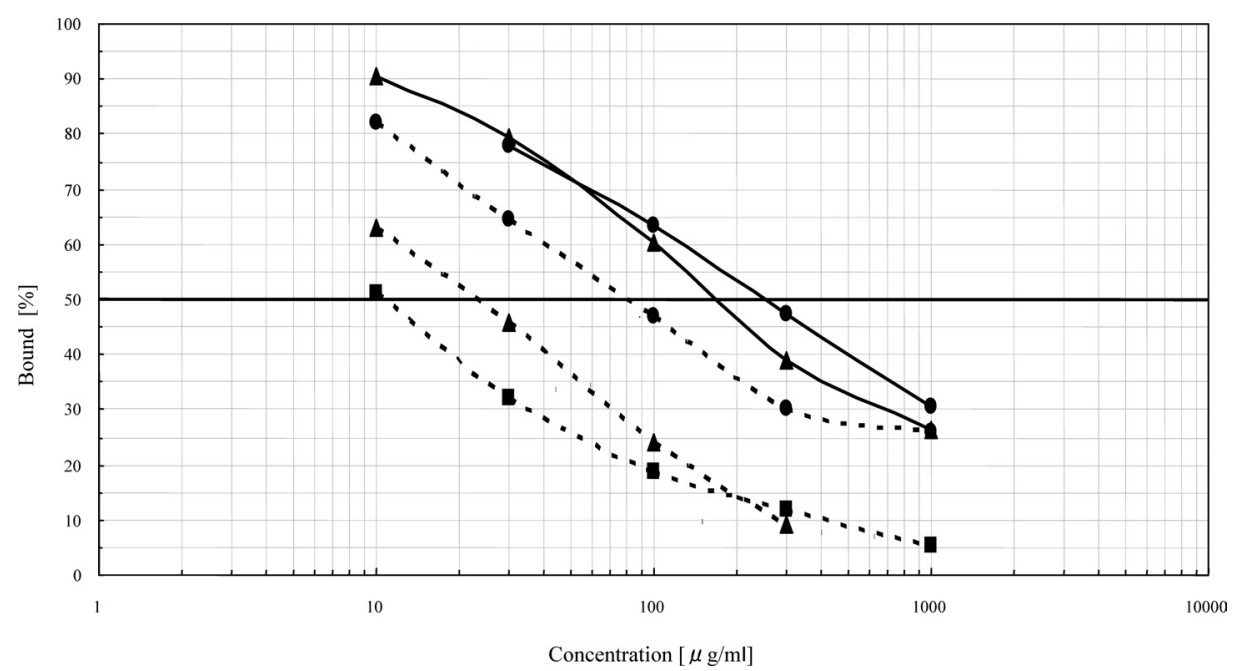

Fig. 3. Inhibition Curve in the ELISA That Was Performed Using a Specific Antibody against the Gelatin

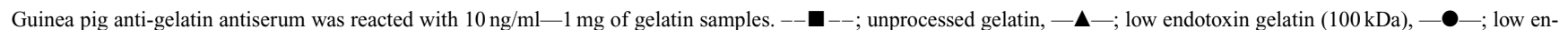
dotoxin gelatin $(50 \mathrm{kDa}),--\boldsymbol{\Delta}_{--}$; unprocessed collagen, $--\mathbf{-}_{--}$; low antigenicity collagen. 

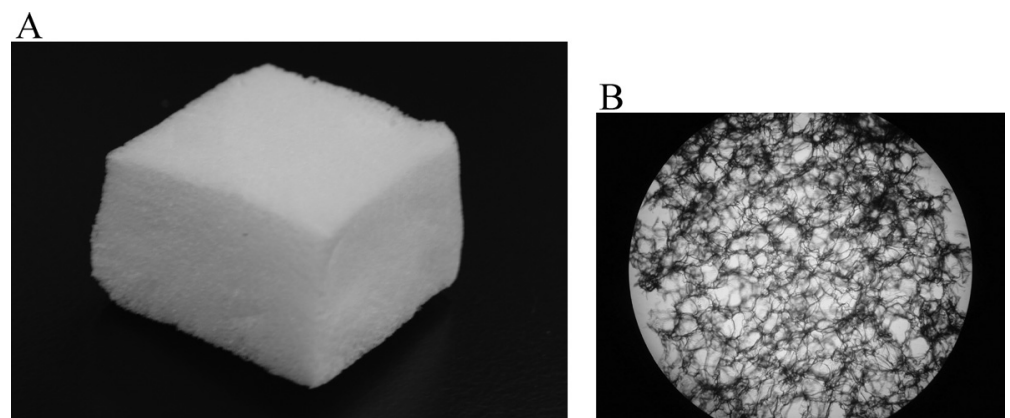

Fig. 4. Sponge Prepared Using Low Endotoxin Gelatin

A; appearance, B; magnified section $(\times 40)$.

pealing for use in tissue culture. We therefore developed a gelatin product that not only met the standards for "purified gelatin" in the Japanese Pharmacopoeia but was of a higher quality because of its low endotoxin content, ability to gel and low antigenicity. Incidentally, the standard for endotoxin in water for injection is below $0.25 \mathrm{EU} / \mathrm{ml}$ and the low endotoxin gelatin produced met this standard even with a concentration of $0.8 \%$ in water demonstrating that the endotoxin levels were safe.

The basic concept of tissue engineering, which is a technology that is commonly applied in regenerative medicine, is coordination between cells, scaffolds and proliferating factors. The coordination with two other elements is an important issue to gelatin that is used as scaffold. Gelatin itself is a protein extracted from collagen that accounts for about a third of the structural proteins of the body and therefore it is compatible with various types of cells. Its compatibility with cell proliferation factors is also considered to be good because there are various types of cell proliferating factors in the extracellular matrix that contain primitive collagen. In addition, the electrical properties of gelatin including low endotoxin gelatin, differs according to the method of extraction from collagen. For example, with alkali-extracted gelatin, the acid amide radical of the collagen side-chain is degraded converting it to a carboxyl radical. This lowers the isoelectric point of the gelatin to around 5.0. On the other hand, with acid-extracted gelatin, the isoelectric point is 9.0 with no such hydrolytic degradation of the side chain. The gelatin used in the present study was acid-extracted gelatin. Thus, there is the possibility that basic cellular growth factors such as bFGF (fibroblast growth factor) and TGF- $\beta 1$ (transforming growth factor) and gelatin gel may be immobilized by coulombic interaction. ${ }^{10)}$ In other words, as the isoelectric point of cellular growth factors in general is higher than 8.5, it may be possible to convert the aqueous solutions of many cellular growth factors to the lyophilized hydroxy gel by dripping, leaving it to stand for a fixed time and the factors may be immobilized in the hydroxy gel mainly by coulombic interaction. In addition, in vivo absorbency may also be controlled depending on the extent of cross-linkage of the gel. ${ }^{11)}$ As such, biodegradable scaffolds with the important property of being able to bind to growth factors are biomaterials that are indicated in regenerative medicine. Moreover, rather than the activity of the bound cellular growth factors being transient, it would be even better if it could be a persistent activity that is slowly released. Use of this low endotoxin gelatin with the ability to gel as scaffold will assist in enhancing the coordination between cells and growth factors. If the medical field also avoids using collagen/gelatin from cows due to effects of bovine spongiform encephalopathy the demand for gelatin derived from non-cow sources will further increase.

Hereafter, we plan to confirm the superiority of low endotoxin gelatins prepared using this method and with various types of cultured cells as biomaterials, and also conduct safety tests on gelatin itself.

\section{REFERENCES}

1) Brock-Utne J. G., Gaffin S. L., Anaesth. Intensive. Care, 17, 49-55 (1989).

2) Ziegler E. J., McCutchan J. A., Fierer J., Glauser M. P., Sadoff J. C., Douglas H., Braude A. I., N. Engl. J. Med., 307, 1225-1230 (1982).

3) Brunson K. W., Nicolson G. L., J. Supramol. Struct., 9, 231-241 (1978).

4) Harlan J. M., Harker L. A., Reidy M. A., Gajdusek C. M., Schwarts S. M., Striker G. E., Lab. Invest., 48, 269-274 (1983).

5) Ongradi J., Kulcsar G., Bertok L., Folia. Microbiol. (Praha), 29, 450 454 (1984).

6) Kirikae T., Tamura H., Hashizume M., Kirikae F., Uemura Y., Tanaka S., Yokochi T., Nakano M., Int. Immunopharmacol., 19, 255-265 (1997).

7) “The Japananese Pharmacopoeia," 15th ed., ed. by Ministry of Health, Labour and Welfare, Hirokawa Publishing Co., Tokyo, 2006, p. 159.

8) Abiko Y., et al., "Glues and Gelatins," 2nd ed., ed. by Gelatin and Glue Manufacturers of Japan, Maruzen Co., Ltd. Pub., Osaka, 1997, pp. $245-288$.

9) "PAGI METHOD," 8th ed., ed. by Commission on Methods for Testing Photographic Gelatin, Tokyo, 1997, pp. 8-11.

10) Tabata Y., Protein Nucleic Acid, Enzyme, 45, 2179-2187 (2000).

11) Tabata Y., Ikada Y., Biomaterials, 20, 2169—2175 (1999). 